\title{
Meta-heurística de busca tabu aplicada em simulação de rede de conexão entre filtros digitais Butterworth
}

\author{
Leonardo G. Aleixo ${ }^{1}$ \\ PPGCC/UFPA, Belém, PA
}

\begin{abstract}
Resumo. Este documento implementa matemática em meta-heurística e simula pontos de conexões ótimas para conjunto de filtros digitais Butteworth, onde as freqüências de valores baixos devem estar no limite da banda de passagem. É conceituada a importância dos filtros digitais para obter melhores valores ótimos de acoplamento entre os mesmos filtros e em rede, apresentando o estudo de caso com análise numérica com o melhor conjunto de soluções em conexão retornadas pelo algoritmo de busca Tabu. Sendo utilizados parâmetros digitais de filtros para a entrada de valores e com processamento, retornando conjunto de pontos para filtrar um sinal transmitido e efetuar controle programável em rede de.telecomunicações.
\end{abstract}

Palavras-chave. Engenharia, Telecomunicações, Meta-heurística, Busca-tabu, Python

\section{Introdução}

Para a engenharia eletrônica é importante poder recorrer a mais de uma opção utilizando modelos matemáticos, onde os mesmos possam ser convertidos em circuitos eletrônicos ou código programável computadorizado. Os projetos em telecomunicações tentam melhorar o equilíbrio entre o baixo custo de equipamentos com uma maior capacidade de transmissão de dados, buscando desenvolver uma rede de comunicação onde a qualidade do sinal possa alcançar grandes distâncias e maior número de equipamentos de recep̧̧ão. Pensando nisso é que este trabalho desenvolveu método alternativo, utilizando meio computacional programável com modelo matemático, simulando resultado numérico dos melhores pontos otimizados de uma rede de filtros digitais, com o retorno de maior potência e melhor ganho de sinal. O método deste projeto pode ser expandido para sistemas embarcados, simulações de defesa estratégica em comunicação digital, e também possibilitar inserir outros tipos variados de modelos de filtros digitais.

\section{Filtros digitais com otimização aplicada}

Com referências a importância do uso de filtros digitais e otimização, seguem as pesquisas que servem de base para o estudo deste projeto de simulação. Vemos que em [13] filtros digitais podem ser deduzidos a partir de modelos de filtros analógicos, para a analise do comportamento de impulsos recebidos em canais de transmissão. O uso de filtros digitais tem também importância no mapeamento da informação em imagens digitais $[1,12,14]$, na programação de computadores em software de análises numéricas e para a criptografia de sinais visto em [8]. Também existem estudos voltados para o suprimento e qualidade da energia elétrica nos centros urbanos, com uso de recursivas para reduzir do Impacto da Resposta Transitória do TPC visto em [15]. Dentro

\footnotetext{
${ }^{1}$ leotelecom@bol.com.br.
} 
do estudo de otimização, temos método [10] baseado em algoritmo da colônia de formigas para a eficiência de projeto de filtros IIR digitais em rápida convergência nos resultados por design. Conforme [11] é aplicado o algoritmo Busca Tabu que impõe regras probabilísticas com estratégias de soluções ótimas, para mínimos locais em tempo razoável para projetar filtro ideal de resposta finita ao impulso (FIR). Para [4] é testado algoritmos de meta-heurística que otimizão filtros digitais de resposta infinita ao impulso (IIR) objetivando minimizar a resposta de superfície de erro. Segundo [3] algoritmos heurísticos não garantem encontrar a solução ótima para um determinado problema porém são capazes de melhorar uma solução existente e retornar uma solução viável e de qualidade em tempo razoável. Em [6] o estudo em Meta-Heurística amplia possibilidades de resposta a eventos utilizando diversos algoritmos, compilando conceitos de otimização na aplicação lógica programável.

\section{Estudo de caso}

O Algoritmo 1 busca Tabu foi aplicado como modelo de meta-heurística e o mesmo foi adaptado ao problema da mochila, o que para este trabalho busca alcançar um esquema de gestão de memória que reduza a possibilidade de examinar mais de uma vez um mesmo conjunto de filtros conectados e configurados em rede simulada(ou solução do problema $\boldsymbol{S}$ ), em que a solução de conexão entre filtros de potência média em $d B m$ e ganho máximo em $d B$ é definida pela programação em código computacional. O conjunto das proibições do algoritmo é registrado em uma memória dinâmica que possibilita sua alteração de acordo com o progresso da busca e demais circunstâncias. A função (1) e função (2) em código Python são encontradas em [2] e aplicadas em conjunto, possibilitando gerar uma função objetivo modelada que representa a equação (3) do filtro digital Butteworth passa-baixa, com sua eficiência ligada a máxima ordem de sua equação polinomial.

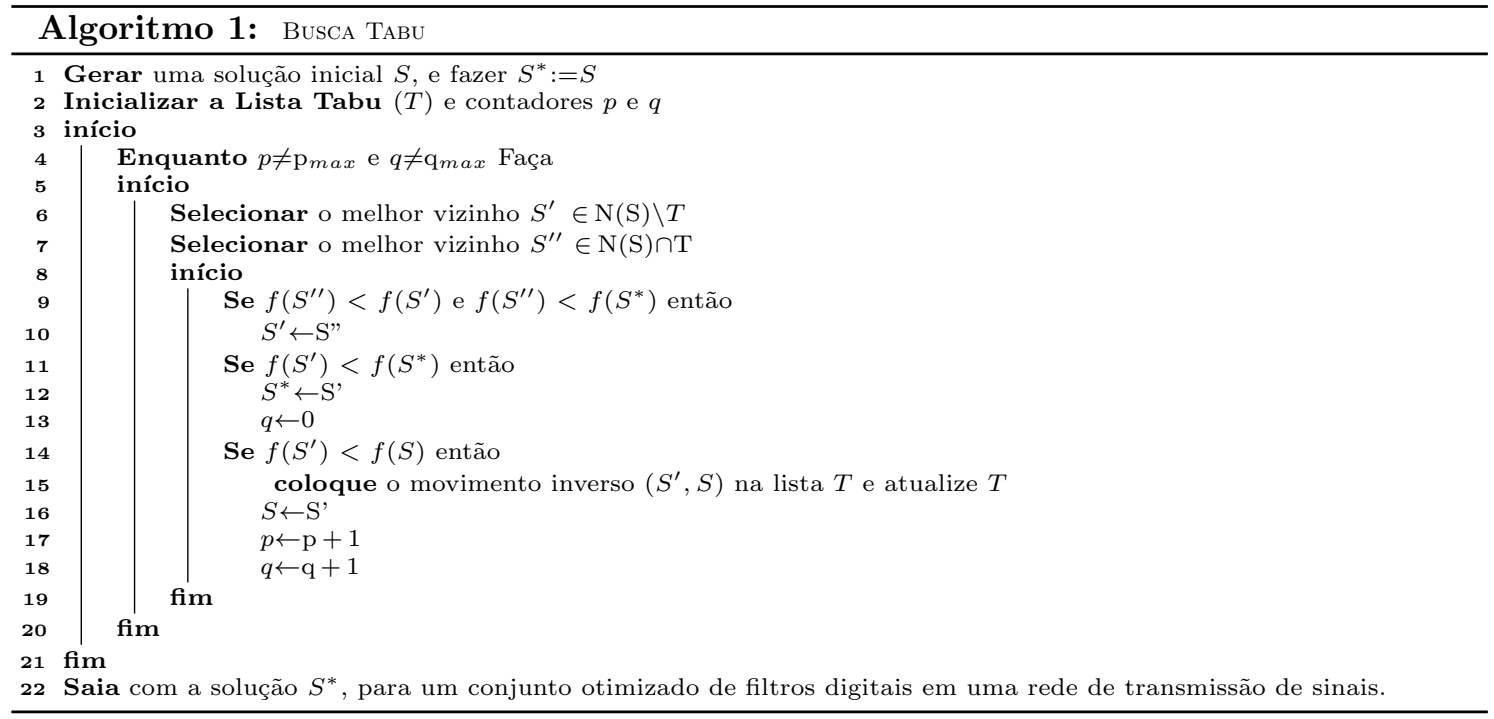

Algumas das vantagens da simulação em computador são, a flexibilidade de alteração do código, a portabilidade para executá-la em equipamentos eletrônicos compatíveis, e principalmente a incorporação de modelos matemáticos. Logo para este trabalho, foi adaptado o código computacional 
escrito em linguagem Python ${ }^{2}$ com o adicionamento das funções de filtros digitais e existentes na biblioteca SciPy ${ }^{3}$. Observamos que em [9] o repositório SciPy(Scientific Computing Tools for Python) contém bibliotecas de código aberto em linguagem Python desenvolvido para matemáticos, cientistas e engenheiros e de livre instalação, fornecendo código fonte das funções de projeto do filtro digital Butteworth, e que permitem manipular dados numéricos através da função $\mathrm{NumPy}$ (Base $\mathrm{N}$-dimensional array package) e utilizada para o processamento de vetores e matrizes de dados, e para o processamento digital de sinal de um filtro. É demonstrado o diagrama simulado de uma rede de conexão Figura 1(a) entre filtros, que analisa soluções viáveis Figura 1(b) para o ganho médio do filtro combinando a ordem da equação do polinômio [5]. Os filtros são unidos com parâmetros de entrada ótimos. O diagrama Figura 1(c) simplifica acoplamento entre dois sistemas lineares invariantes com o tempo (LIT), representando a passagem de sinal digital por entre dois filtros, e demonstrando ligação entre filtros de resposta discreta.

Cada projeto pode ser definido como $\left(\mathbf{P}_{n}, \forall n \mid n \in \mathbb{N}: n \geq 1\right)$ para um filtro digital que restringe freqüências superiores a da freqüência de corte, incorporando os seguintes parâmetros de retorno: (N) ordem do filtro, (Wn) freqüência de corte e (k) ganho do filtro; Temos também os parâmetros de entrada de projeto, (wp) freqüência de borda de passagem, (ws) freqüência de faixa de passagem, (gpass) perda máxima na banda passante e (gstop) atenuação mínima na banda de parada, tendo (low) que define tipo passa baixa de filtro e (False) que especifica análise digital dos parâmetros de entrada.

$$
\begin{gathered}
\mathbf{N}, \mathbf{W n}=\text { signal.buttord }(w p, \text { ws, gpass, gstop, False }) . \\
\mathbf{K}=\text { signal.butter }\left(N, \text { Wn }, \text { low }, \text { False },{ }^{\prime} k^{\prime}\right) . \\
\mathbf{P}_{n}=(1) \cup(2)=\{\forall(n, N, K) \in \mathbb{N}: \forall(W n, w p, w s) \in \mathbb{R}\} \\
\left.H[Z]\right|_{Z=e^{j \Omega}}=\sum_{k=1}^{N} \frac{T_{d} A_{k}}{1-e^{s_{k} T_{d}} Z^{-1}}
\end{gathered}
$$

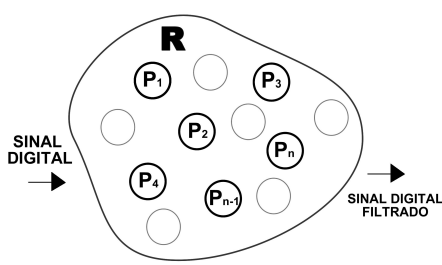

(a)

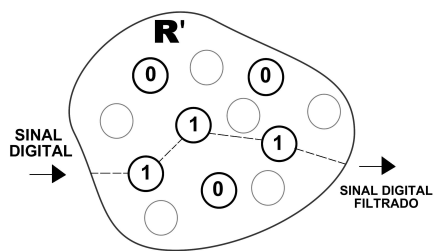

(b)

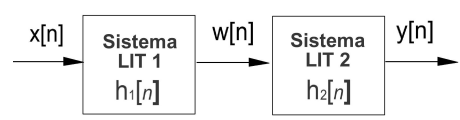

(c)

Figura 1:

Figura 1: Em (a) vemos rede $\mathbf{R}=\left\{\mathbf{P}_{n} \mid \forall n \in \mathbb{N}: n \geq 1\right\}$ de filtros digitais. Para (b) temos rede $\mathbf{R}^{\prime}=\left\{\mathbf{P}_{n}=\{0,1\} \mid \forall n \in \mathbb{N}: n \geq 1\right\}$ onde 1 indica um filtro viável na rede para ser conectado. Vemos em (c) casamento de dois sistemas lineares invariantes com o tempo, onde $\mathbf{x}[\mathbf{n}]$ é o sinal original de entrada na rede, o w[n] é o primeiro sinal filtrado, e o $\mathbf{y}[\mathbf{n}]$ é o sinal filtrado de saída da rede.

\footnotetext{
${ }^{2}$ https://github.com/marcoscastro/mochila ${ }_{i}$ nteiro - busca $_{t} a b u$

${ }^{3}$ https://docs.scipy.org/doc/scipy/reference/signal.html
} 


\subsection{Desenvolvimento da função otimizada}

Aqui é definida função que receberá dados numéricos de resposta para os filtros Butteworth, apresentando o modelo matemático que vai otimizar melhores soluções de conexão. O parâmetro K vai receber o valor que limita o ganho médio esperado e decidindo quais projetos de filtros podem ser conectados em rede de transmissão. Conforme o algoritmo busca Tabu traga as soluções ótimas o valor do ganho máximo pode ultrapassar os $32 \mathrm{~dB}$, mesmo retornando filtros viáveis de conexão através dos outros parâmetros digitais. Se o ganho for muito maior do valor definido como limite, pode vir a ser um fator no aumento dos custos na elaboração e produção de componentes eletrônicos, que farão parte de uma rede de telecomunicações. Logo, vemos a função objetivo com modelo maximamente ajustado, para fazer a busca de todos os filtros com melhor solução interligada, e limitada ao $\mathbf{K}_{\text {máximo }}$.

$$
\begin{array}{ll}
\text { Variável de decisão } & : \mathbf{A}_{i}, \text { onde } i=1,2, \cdots, 10 \\
\text { Restrição para para a potência média } & : 0 \mathrm{dBm} \leq \mathbf{A}_{i}<\approx-200 \mathrm{dBm} \\
\text { Função objetivo para solução ótima } & : \quad S_{n}=\left[\prod_{j=0}^{V}(\text { peso })\right] \cdot\left[\text { Máximo }\left(\sum_{i=1}^{V} \operatorname{solucão}[j] \cdot \mathbf{A}_{i}\right)-\mathbf{A}_{\text {máximo }}\right]
\end{array}
$$

\subsection{Aplicação da meta-heurística}

Em [7] o projeto de filtros é normalmente realizado no domínio da freqüência e o filtro Butterworth tem resposta de banda passante, máxima plana, adequada para um sinal de pequena distorção e também sendo filtro de montagem simples, por essas características foi escolhido para este trabalho. A instância gerada para este trabalho utilizou como base um vetor, onde o mesmo conteve 10 amostras de projetos visto na Tabela 1 com cada projeto apresentando quatro parâmetros de entrada (wp,ws,gpass,gstop) e dois parâmetros de retorno ( $\mathbf{W n}, \mathbf{N}, \mathbf{K})$, gerando conjunto de dados de até 60 valores a serem analisados, no entanto os parâmetros de entrada em freqüência tiveram que ser normalizados para a taxa de freqüência e leitura de amostras de $5000 \mathrm{~Hz}$, em conformidade ao Teorema da amostragem (Nyquist ${ }^{4}$ e Shannon ${ }^{5}$ ).

\subsection{Resposta numérica e análise}

O processamento do algoritmo utilizou ganho máximo $32 d B$, imposto como limite médio para o conjunto de filtros e combinado a ordem $\mathbf{N}$ que define o melhor coeficiente para o conjunto de equações polinomiais dos filtros, e assim definindo melhor qualidade de resposta ao sinal amostrado. Sete soluções ótimas são obtidas pelo algoritmo aplicado, e são apresentadas na Tabela 2, onde se constata que os projetos $P_{4}$ e $P_{8}$ são mais freqüentes nas setes soluções e a sexta solução possui maior incidência de filtros otimizados. Vemos que na Tabela 3 a própria sexta solução apresenta valores médios de $(4,70)$ onde o valor 4 é a ordem $\left(\mathbf{N}_{\text {media }}\right)$ e o valor 70 é o ganho $\left(\mathbf{G}_{\text {medio }}\right)$. Podese também representar o polinômio equivalente do conjunto de filtros da solução 6 . O retorno das soluções ótimas são apresentadas no formato de valores binários $[0,1]$ para simplificar a resposta de soluções.

\footnotetext{
${ }^{4}$ Harry Theodor Nyqvist - foi um engenheiro eletrônico nascido na Suécia.

${ }^{5}$ Claude Elwood Shannon- foi um matemático, engenheiro eletrônico e criptógrafo estadunidense.
} 
Tabela 1: Amostras de $P_{1}$ a $P_{10}$, para o conjunto de 10 iterações.

\begin{tabular}{|c|c|c|c|c|}
\hline $\begin{array}{c}\text { Filtro } \\
\left(P_{n}\right)\end{array}$ & $\begin{array}{l}\text { Frequiência }(\mathrm{Hz}) \text { - }[\mathrm{wp}] \\
\text { de borda de passagem }\end{array}$ & $\begin{array}{l}\text { Frequêencia }(\mathrm{Hz})-[\mathrm{ws}] \\
\text { de faixa de passagem }\end{array}$ & $\begin{array}{c}\text { Perda máxima (dB) - [gpass] } \\
\text { na banda passante }\end{array}$ & $\begin{array}{c}\text { Atenuação mínima (dB) - [gstop] } \\
\text { na banda de parada }\end{array}$ \\
\hline$\frac{\left.I_{n}\right)}{P_{1}}$ & 100 & 300 & & 12 \\
\hline$P_{2}$ & 150 & 350 & 5 & 15 \\
\hline$P_{3}$ & 200 & 400 & 3 & 12 \\
\hline$P_{4}$ & 250 & 450 & 3 & 18 \\
\hline$P_{5}$ & 300 & 500 & 4 & 10 \\
\hline$P_{6}$ & 350 & 550 & 6 & 13 \\
\hline$P_{7}$ & 400 & 600 & 3 & 15 \\
\hline$P_{8}$ & 450 & 650 & 6 & 14 \\
\hline $\begin{array}{l}P_{9} \\
P_{10}\end{array}$ & 550 & 750 & $\begin{array}{l}2 \\
2\end{array}$ & 16 \\
\hline
\end{tabular}

Tabela 2: Soluções ótimas de conexões $P_{n}$ filtros

\begin{tabular}{|c|c|c|c|c|c|c|c|c|c|c|c|}
\hline Solução & $P_{1}$ & $P_{2}$ & $P_{3}$ & $P_{4}$ & $P_{5}$ & $P_{6}$ & $P_{7}$ & $P_{8}$ & $P_{9}$ & $P_{10}$ & TOTAL \\
\hline 1 & 0 & 1 & 0 & 0 & 1 & 0 & 0 & 0 & 0 & 1 & 3 \\
\hline 2 & 0 & 0 & 0 & 0 & 1 & 0 & 0 & 1 & 1 & 0 & 3 \\
\hline 3 & 0 & 1 & 1 & 1 & 0 & 0 & 0 & 1 & 0 & 0 & 4 \\
\hline 4 & 0 & 0 & 0 & 1 & 0 & 0 & 1 & 0 & 1 & 1 & 4 \\
\hline 5 & 0 & 0 & 0 & 1 & 0 & 0 & 1 & 1 & 0 & 1 & 4 \\
\hline 6 & 0 & 1 & 1 & 1 & 0 & 0 & 1 & 1 & 1 & 0 & 6 \\
\hline 7 & 0 & 0 & 0 & 1 & 0 & 0 & 1 & 1 & 1 & 0 & 4 \\
\hline
\end{tabular}

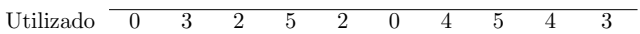

Tabela 3: Conjunto de soluções para (Ordem,Ganho) de filtros

\begin{tabular}{|c|c|c|c|c|c|c|c|c|c|c|c|}
\hline Solução & $P_{1}$ & $P_{2}$ & $P_{3}$ & $P_{4}$ & $P_{5}$ & $P_{6}$ & $P_{7}$ & $P_{8}$ & $P_{9}$ & $P_{10}$ & Média \\
\hline 1 & 0 & $(2,48)$ & 0 & 0 & $(2,41)$ & 0 & 0 & 0 & 0 & $(7,122)$ & $(4,34)$ \\
\hline 2 & 0 & 0 & 0 & 0 & $(2,41)$ & 0 & 0 & $(3,58)$ & $(5,89)$ & 0 & $(3,63)$ \\
\hline 3 & 0 & $(2,48)$ & $(2,44)$ & $(4,84)$ & 0 & 0 & 0 & $(3,58)$ & 0 & 0 & $(3,59)$ \\
\hline 4 & 0 & 0 & 0 & $(4,84)$ & 0 & 0 & $(5,95)$ & 0 & $(5,89)$ & $(7,122)$ & $(5,70)$ \\
\hline 5 & 0 & 0 & 0 & $(4,84)$ & 0 & 0 & $(5,95)$ & $(3,58)$ & 0 & $(7,122)$ & $(5,62)$ \\
\hline 6 & 0 & $(2,48)$ & $(2,44)$ & $(4,84)$ & 0 & 0 & $(5,95)$ & $(3,58)$ & $(5,89)$ & 0 & $(4,70)$ \\
\hline 7 & 0 & 0 & 0 & $(4,84)$ & 0 & 0 & $(5,95)$ & $(3,58)$ & $(5,89)$ & 0 & $(4,82)$ \\
\hline
\end{tabular}

\section{Rede simulada com otimização de filtros conectados}

Após a obtenção dos resultados numéricos por simulação computacional meta-heurística, vemos a convergência de resultados por iterações de dados de amostragem dos filtros. Foram obtidas novas soluções para um conjunto maior de filtros de uma rede simulada, que para efeito de projeto, utilizou valores numéricos aplicados em projetos de filtros digitais. É importante frisar que os coeficientes numéricos de projetos de circuitos eletrônicos, provem de componentes reais acoplados, para processar o sinal de entrada e retornar sinal de saída maximamente previsível. Em telecomunicações, a busca por equilíbrio orçamentário e tecnológico leva a estudos de viabilidade, para melhorar o ganho de um sinal transmitido por um canal de transmissão, e que exija menor conjunto de circuitos acoplados. Através desse método, torna-se possível desenvolver aplicações em sistemas móveis de transmissão a até grandes softwares de engenharia, para o usuário comum e até a grande indústria. Novos modelos de filtros digitais e analógicos podem ser avaliados em uma rede de comunicação simulada, com redução de ruídos e interferência, para que o software possa, em tempo razoável, decidir qual melhor conjunto de conexão de filtros em uma rede de telecomunicações. Vemos que a Figura 2 apresenta diagrama para proposra de possível rede de conexão para o conjunto de filtros digitais. 


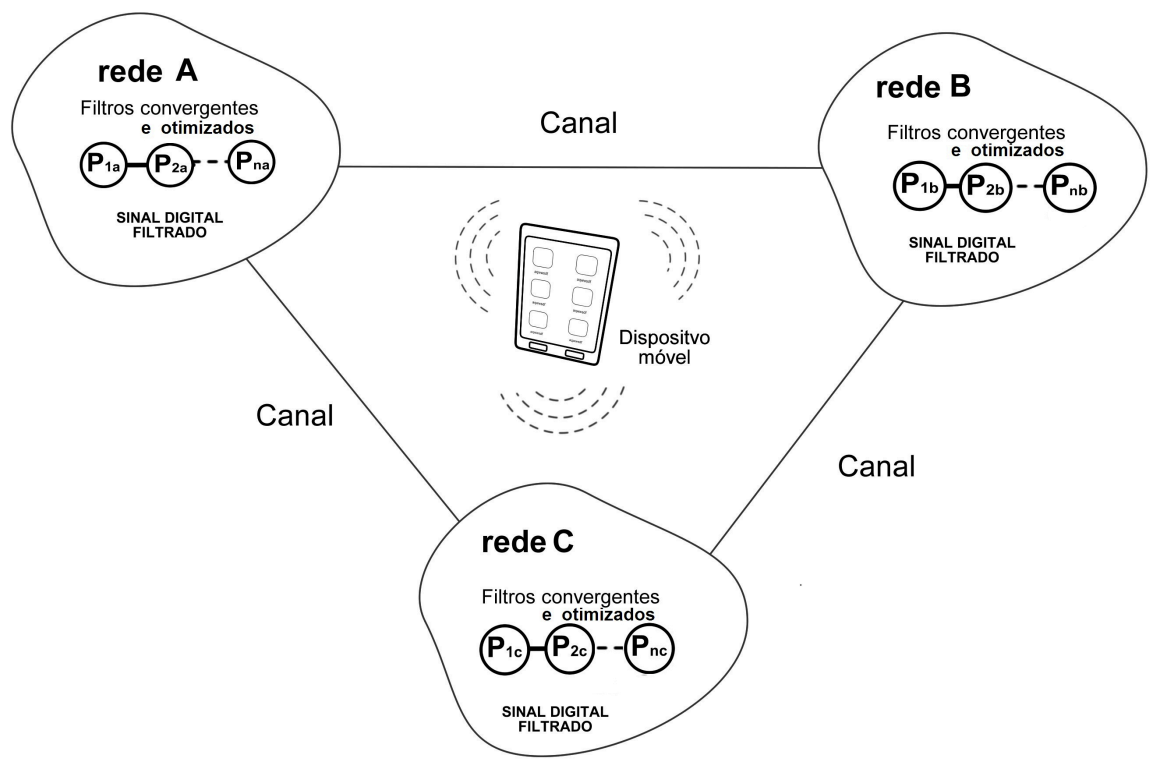

Figura 2:

Figura 2: A rede $\mathbf{A} \subset\left[P_{1 a}, . ., P_{n a}\right]$ está conectada a rede $\mathbf{B} \subset\left[P_{1 b}, . ., P_{n b}\right]$ e a rede $\mathbf{C} \subset$ $\left[P_{1 c}, . ., P_{n c}\right]$, onde $P_{n}$ são projetos de filtros digitais convergentes obtidos por algoritmo de soluções ótimas.

\section{Conclusão}

Os valores numéricos utilizados, considerando as fontes em que foram aplicados, devem estar dentro do comportamento estável e rigor técnico de transmissão. Vemos que a análise final de soluções otimizadas retornou o comportamento simulado de uma rede de transmissão, para os valores de respostas do filtro digital. Ao aplicar a meta-heuristica Busca-Tabu ao modelo matemático determinístico de filtro digital se obteve resultados de iterações, com instâncias de valores próximos ao estudo de projetos acadêmicos em modelagem de filtros. No entanto, um projeto real para utilizar modelos matemáticos de circuitos eletroeletrônicos completos, deve requerer estudos amplos de eletrônica e valores de entrada reais, compatíveis ao filtro utilizado no equipamento de transmissão de sinais. Este trabalho mostra possibilidade de desenvolvimento de aplicação profissional, que amplie o algoritmo utilizado, onde o mesmo possa ser incorporado em software em sistemas embarcados voltados para a transmissão de sinal digital, em redes VPN, LAN, MAN, RAN, WAN e telecomunicações. Como visto, não foram aprofundados estudos matemáticos para criar nova meta-heuristica, mas somente adaptando a mesma ao modelo de filtro digital com método de otimização, combinando estudos de engenharia de transmissão de sinal com modelagem matemática aplicada. Também temos um ensaio teórico que busca base para desenvolver sistemas embarcados elaborados, controlando e redirecionando um vetor de transmissão de sinais em uma rede de telecomunicações. 


\section{Referências}

[1] Angelo, N. P. Aplicação de filtros de Gabor no processo de classificação de imagens digitais com base em atributos de textura, Dissertação de Mestrado, UFRGS, 2000.

[2] Butter, S. Scientific Computing Tools for Python, https://docs.scipy.org/doc/scipy/reference/ generated/scipy.signal.butter.html. English, 2018.

[3] Da Silva, A. P., \& de Oliveira Nunes, É. Busca tabu e o problema de roteamento de veículos: Uma aplicação prática no Exército Brasileiro, RICAM-Revista Interdisciplinar de Ciências Aplicadas à Atividade Militar, volume 2, número 2, páginas 119-134, 2008.

[4] Dai, C., Chen, W., \& Zhu, Y. Seeker optimization algorithm for digital IIR filter design, IEEE transactions on industrial electronics, volume 57, número 5, páginas 1710-1718, 2009. DOI: 10.1109/TIE.2009.2031194.

[5] Dorf, R. C., \& Svoboda, J. A. Introdução aos circuitos elétricos, $8^{a}$ edição. Grupo Gen-LTC, Rio de janeiro, 2012.

[6] Goldbarg, E., Goldbarg, M., \& Luna, H. Otimização Combinatória e Metaheurísticas: Algoritmos e Apliacações, $1^{\underline{a}}$ edição. Elsevier Brasil, Rio de Janeiro, 2016.

[7] Haykin, S., \& Moher, M. Sistemas de Comunicação, 5⿳a edição. Bookman, Porto Alegre, 2011.

[8] Jascone, F. L. T. Protótipo de software para ocultar texto criptografado em imagens digitais, Trabalho de Conclusao de Curso, p. 64, FURB, 2003.

[9] Jones, E., Oliphant, T., \& Peterson, P. SciPy: Open source scientific tools for Python, https://www.scipy.org, 2001.

[10] Kalinli, A., \& Karaboga, N. Artificial immune algorithm for IIR filter design, Engineering Applications of Artificial Intelligence, volume 18, número 8, páginas 919-929, 2005, https://doi.org/10.1016/j.engappai.2005.03.009.

[11] Karaboga, D. E. R. V. Í. S., Horrocks, D. H., Karaboga, N. U. R. H. A. N., \& Kalinli, A. D. E. M. Designing digital FIR filters using Tabu search algorithm, IEEE International Symposium on Circuits and Systems (ISCAS), volume 4, páginas 2236-2239, IEEE, junho 1997. DOI: 10.1109/ISCAS.1997.612766.

[12] Niemann, R. S. Comparação de métodos de filtragem e geração de modelos digitais de terreno a partir de imagens obtidas por veículo aéreo não-tripulado, Dissertação de Mestrado, Unesp, 2017.

[13] Oppenheim, A. V., \& Schafer, R. W. Processamento em tempo discreto de sinais, $3^{a}$ edição. Pearson Education do Brasil, São paulo, 2012.

[14] Rocha, L. A. Formas de interação entre humanos e dados digitais em ambientes virtuais, Horizontes Antropológicos, volume 10, número 21, páginas 155-179, 2004, https://doi.org/10.1590/S0104-71832004000100007.

[15] Silva, C. A. D. Filtros digitais recursivos para redução do impacto da resposta transitória do TPC, Tese de Doutorado, UFCG, 2014.

[16] Silva, L. A. P., \& de Assis Brito Filho, F. Sistema de filtragem digital reconfigurável baseado em FPGA para aplicação em rádio definido por software, edição E29, Revista Ibérica de Sistemas e Tecnologias de Informação, http://www.risti.xyz/issues/ristie29.pdf, páginas 299311, 2020. ISSN: 16469895. 